\title{
The Impact of Small-Scale Irrigation on Household Food Security: The Case of SNNP of Eth the Impact of Small-scale Irrigation on Household Food Security: The Case of SNNP of Ethiopia
}

\author{
Musba Kedir
}

\begin{abstract}
Even though Ethiopia is endowed with natural resource, it is one of the poorest country in world. The reason for this is maluses of the natural resource and dependence on traditional and rain feed agriculture. Small scale irrigation is applied in Ethiopia more than 5 decades and in SNNP region 3 decades ago. This study was conducted in SNNP region with 60 small scale irrigation users and 343 non users. The objective of this study was to evaluate the impact of small scale irrigation on household on food security. To analyze the data descriptive statistics like chi2 for categorical variables and t-test continuous predictor variables was used. Propensity Score matching model was used to analyze the impact of the small scale irrigation on food security of the house hold. Based on this study application of small scale irrigation in the study area does not contribute to be food secured. This may be due to the region is getting high rainfall relative to other regions like Afar, Somali and Tigray region. The farmers does not want to west their time on irrigation activities doe to plenty of rainfall and precipitation. On the other hand, most of the farmers staple food is depend on Inset (falls banana) which does not need irrigation. Based on this study we recommend that, irrigation activities should be applied in areas by which rainfall is scarce like the regions Tigray, Afar and Somali region by which rain is scarce.
\end{abstract}

Keywords: food index, Irrigation, PSM model, SNNP region

DOI: $10.7176 /$ ISDE/12-1-02

Publication date: January $31^{\text {st }} 2021$

\section{Irrigation}

Ethiopia is one of the poorest countries in the world, where about $29.2 \%$ of its population live below poverty line (World Bank, 2013). Most of the Ethiopian population lives in rural areas and the livelihood of the greater majority of this is based on rain fed agriculture that is subject to highly irregular rainfall pattern with detrimental impact on agricultural production.

Moreover, agriculture accounts for over $40 \%$ to the GDP, out of which $95 \%$ of the production comes from smallholder farmers (MoARD, 2010).

Dependence on natural factors of production as well as small and fragmented holdings, environmental degradation, rapid population growth, low access to new agricultural technologies, traditional methods of cultivation, and low institutional support are identified as factors that keep smallholder production at subsistence level in the country (MoFED, 2012). To address these issues, Agricultural Development Led Industrialization (ADLI) Strategy was designed in 1991 where focus was given to the expansion of small scale irrigation, formation of cooperative societies and access to agricultural technologies to answer the food demand and bring about the socioeconomic development in the country. Irrigation is one of the agricultural technologies defined as the man made application of water to guarantee double cropping as well as steady supply of water in areas where rainfall is unreliable. Hence, the development of small-scale irrigation is one of the major interventions to increase agricultural production in the rural parts of the country.

Ethiopia's irrigation potential is estimated at 3.7 million hectare, of which only about 190,000 ha ( $4.3 \%$ of the potential) is actually irrigated. There is little information on the extent to which the so far developed irrigation schemes have been effective in meeting their stated objectives of attaining food self-sufficiency and eradicating poverty.

The country endowed by water resources. The country is divided into 12 basins. Moreover, the country is water tower of the Horn of Africa. But, the potential is not fully utilized because of many challenges including limited financial resources, technical challenges, improper or inefficient irrigation water delivery performance, unavailability of irrigation scheme performance, dissatisfaction of irrigation user, and lack of good governance. Furthermore, for the country Ethiopia, irrigation agriculture comes in bold on enhance agricultural productivity and poverty reduction.

Livelihoods of the rural people of the country depend on agriculture. However, erratic nature of rain and prevalence of drought in the country make agricultural production a challenge. To counter this problem, use of the available water resource for irrigation development is the most promising option.

Even though different studies are undertaken on impact of irrigation on food security of the households' heads in Southern Nations Nationalities and Peoples of Ethiopia, the study only depends on specific irrigation Schemes. 
This study focuses on the major parts of different irrigations schemes applied in SNNP regional state of Ethiopia.

\section{Objectives of the study}

The general objective of this study is to examine the impact of small scale irrigation on the Household food security of the users in SNNP regional state.

The specific objectives of this study are:

1. To identify the determinant factors that affects the household food security.

2. To analyze the impact of irrigation on food security.

\section{Method of data analysis}

The study employed both descriptive and econometric techniques. The descriptive analysis was performed using frequencies, means, and maximum and minimum values. The econometric analysis employed the Propensity score matching (PSM) to identify the impact of small scale irrigation on household food security from among possible other household food security influencing factors.

\section{Propensity Score Matching}

For more than two decades, advanced statistical methods known as propensity score (PS) techniques, have been available to aid in the evaluation of cause-effect hypotheses in observational studies. In many fields of the social sciences, there is a growing interest in methods that can be used to evaluate the effects of social programs and public policies. A large part of the recent literature on program evaluation focuses on estimation of the average effect of the treatment under the potential outcomes framework for causal inference, which was pioneered by Neyman (1923) and Fisher (1925) and extended by Rubin (1974, 1978) to observational studies. Following the seminal work of Rosenbaum and Rubin (1983a) the literature on estimating average treatment effects under the unconfoundedness assumption has become widespread (Imbens, 2004).

\section{The propensity score matching methodology for the estimation of causal effects}

We use a standard setup in the treatment effect literature. Let us suppose we have a population of individual units under study indexed by $\mathrm{i}=1,2, \ldots, \mathrm{N}$, an indicator for a binary treatment, $\mathrm{T}$, which assumes the value 1 for treated units and 0 for untreated, or controls, and an outcome variable, which we indicate by $Y$. Under the potential outcomes framework, each unit, $i$, has two potential outcomes associated with the two treatment levels: Yi1 if Ti $=1$ and $\mathrm{Yi} 0$ if $\mathrm{Ti}=0$. Potential outcomes for unit $\mathrm{i}$ and treatment $\mathrm{t}$ can be written as $\mathrm{Yid}$, with $\mathrm{t}=\{0,1\}$. The fact that this variable is labelled only by $\mathrm{i}$ and $\mathrm{t}$ corresponds to the "no interference among units" assumption of Cox (1958), which Rubin (1980) extended to the Stable Unit Treatment Value Assumption (SUTVA). This standard assumption requires that potential outcomes for a unit are not affected by the treatment received by other units and there are no versions of the treatment. Under the SUTVA, we can define several causal effects the most popular being the Average Treatment effect on the Treated:

$A T T=E\left(Y_{1}-Y_{o} / T=1\right)$

Which focuses explicitly on the effects on those for whom the program is actually intended. In particular, the ATT gives the expected effect of the treatment on a randomly drawn unit from the population of treated. It is therefore more interesting for policy makers than the average treatment effect on the whole population (Heckman et al, 1997). The identifying assumptions are usually stated as follows:

Unconfoundedness (A.1): $\quad \mathrm{Y} 1, \mathrm{Y} 0 \perp \mathrm{T} \mid \mathrm{X}$,

Common support (A.2): $\quad 0<\mathrm{P}(\mathrm{T}=1 \mid \mathrm{X})<1$,

Where $\perp$ in the notation introduced by Dawid (1979) means independence. The combination of the two assumptions A.1 and A.2 is referred to as strong ignorability (Rosenbaum and Rubin, 1983a). Assumption A.1, known as the unconfoundedness assumption, asserts that the probability of assignment to a treatment does not depend on the potential outcomes conditional on observed covariates. In other words, within subpopulations defined by values of the covariates, we have random assignment. This assumption rules out the role of the unobservable variables and therefore is referred to also as selection on observables (Imbens, 2004). Assumption A.2, known as the common support assumption, implies equality in the support of $\mathrm{X}$ in the two groups of treated and controls (i.e. Support $(X \mid \mathrm{T}=1)=$ Support $(\mathrm{X} \mid \mathrm{T}=0)$ ) which guaranties that the ATT is well defined (Heckman et al, 1997); otherwise, for some values of the covariates there would be some treated for which we could not find any comparable units in the control group.

It is instructive to remember the decomposition of the selection bias proposed by Heckman et al (1998). They showed that the selection bias (B) can be decomposed in three components: $\mathrm{B}=\mathrm{B} 1+\mathrm{B} 2+\mathrm{B} 3$. The first component, $\mathrm{B} 1$, refers to the bias caused by non-overlapping supports of $\mathrm{X}$ in the treated and control group. The term B2 depends on misweighting within the common support, as the empirical distributions of treated and non-treated may not be the same even when restricted to the common support. Finally, the term B3 is the "true econometric selection bias" resulting from "selection on unobservable", that is, it is the bias arising from a different distribution 
of relevant unobserved variables between treated and controls. Under A.1 the term B3 is zero. The other bias components are cancelled out when we restrict the analysis on the common support (B1) and we balance covariates in the group of treated and control units (B2). Several methods are available to balance covariates across the groups of treated and controls. Among them matching has become very popular. Matching is an intuitive and appealing method, which basic idea consists of contrasting treated and control units with the same characteristics X. Starting from assumption A.1, the basic idea is that within each cell defined by the values of the covariate $\mathrm{X}$ assignment to treatment or control group is random. Therefore, if in a given application we are willing to assume that all relevant variables that affect the selection on treatment and outcome are collected in the set X (and hence we are confident that assumption A.1 holds) we can match each treated unit with one (or more) control unit with the same values of $\mathrm{X}$. The group of treated and matched controls will differ only for the exposure to treatment and, therefore, differences in the outcome between the two groups can be attributed to the treatment. When the number of matching variables is large and/or when some of $\mathrm{X}$ are continuous exact matching becomes unfeasible and a distance metric have to be used to weight comparisons of matched treated and control units. An alternative is to implement the matching on a univariate variable, which "summarizes" the information incorporated in X, as opposed to matching directly on the multivariate set X. Well known are matching methods that use the propensity score, which can be defined as the conditional probability of receiving a treatment given pre-treatment characteristics:

$\mathrm{E}(\mathrm{X}) \equiv \operatorname{Pr}\{\mathrm{T}=1 \mid \mathrm{X}\}=\mathrm{E}\{\mathrm{T} \mid \mathrm{X}\}$.

The substitution of the multivariate set $\mathrm{X}$ with the univariate $\mathrm{e}(\mathrm{X})$ in the matching procedure is justified by two important theorems due to Rosenbaum and Rubin (1983a). The first one, referred to as the balancing property of the propensity score, asserts that conditioning on the propensity score, $\mathrm{X}$ and $\mathrm{T}$ are independent: $\mathrm{X} \perp \mathrm{T} \mid \mathrm{e}(\mathrm{X})$. This result implies that observations with the same propensity score have the same distribution of characteristics $\mathrm{X}$, independently of treatment status. When the propensity scores are balanced across the treatment and control groups, the distribution of all the covariates are balanced in expectation across the two groups. Therefore, matching on the propensity score is equivalent of matching on $\mathrm{X}$. The second theorem shows that if treatment assignment is strongly ignorable given $X$, then it is strongly ignorable given any balancing score, then adjusting for $\mathrm{e}(\mathrm{X})$ is sufficient to produce unbiased estimates of the ATT. On the basis of these two theorems we can write the ATT as: $A T T=E$

$$
e(x) T=1\left[\left(\frac{E}{T}=1, e(X)-{ }^{Y_{O}} /{ }_{T}=0,(e(X))\right)\right]
$$

Where the outer expectation is over the distribution of $\mathrm{e}(\mathrm{X})$ in the sub-population of the treated units. In observational studies the propensity score is not known and it has to be estimated from the data available. Using the common logit or probit models, we can write $\mathrm{e}(\mathrm{X}) \equiv \operatorname{Pr}\{\mathrm{T}=1 \mid \mathrm{X}\}=\mathrm{F}[\mathrm{h}(\mathrm{X})]$, where $\mathrm{F}($.$) is, respectively, the$ normal or the logistic cumulative distribution and $h(X)$ is a function of covariates with linear and higher order terms. The choice of which higher order terms to include, as well as interactions among covariates, is determined solely by the need to balance covariates distribution in the two treatment groups (Dehejia and Wahba, 1999). Simple parametric specifications for the propensity score have indeed often been found to be quite effective in achieving the balancing required (see for example Zhao, 2005). The estimation of the propensity score is, however, not sufficient to estimate ATT using the (3). The reason is that the probability of observing a treated and a control unit with exactly the same value of the propensity score is, in principle, zero, since e $(X)$ it is a continuous variable. Then, we need to use some algorithm to match treated and controls. Various matching methods have been proposed in the literature to overcome this problem and the most widely used are nearest neighbor, stratification, radius, kernel matching (Caliendo and Kopeining, 2008).

\section{Result and Discussion Sampling techniques}

This study used a multistage sampling technique to select the sample respondents. In the first stage, SNNP regional state was selected purposively for the wider use of small scale irrigation. In the second stage, 6 zones were selected randomly. From the selected zones we have selected 2 from each. Finally, the entire list of households including irrigation users and non-users in each district is prepared separately. This is the sampling frame from which the final list of households was selected, using a random sampling technique. Based on this sample frame, 60 users of irrigation and 343 non-user households were selected using proportionate to sample size for a face-to-face interview.

\section{Descriptive study}

\section{Description of the Sampled Household Characteristics}

A combination of different descriptive, the means, inferential, the t-test and $X^{2}$-test, statistics for predictor variables of samples were done on the household level data to inform the subsequent empirical data analysis.

The descriptive and inferential results presented on Table 3 show that there was statistically significant difference between users of irrigation and non-user in terms of TLU, amount of meals used by adults and children 
per day as well as the average use meal by all households at $1 \%$ significant level for all except the average use meal by all households which significant at $5 \%$.

Table 1. Description of continuous variables

\begin{tabular}{lllll}
\hline Variables & \multicolumn{2}{c}{ Mean across adoption categories } & t-test & \multirow{2}{*}{ P-Value } \\
\cline { 2 - 3 } & User & Non-user & & \\
\hline Age of household head & 43.73 & 52.32 & 4.4472 & 0.0000 \\
Livestock holding(TLU) & 4.20 & 1.35 & 9.8184 & $0.0000^{* * *}$ \\
Family size & 5.88 & 6.00 & 0.3473 & 0.7285 \\
Months in food shortage & 4.11 & 3.60 & 1.2540 & 0.2106 \\
Adult no meals per day & 2.10 & 1.92 & 2.7220 & $0.0068^{* * *}$ \\
Child no meals per day & 2.63 & 2.39 & 2.8393 & $0.0048^{* * *}$ \\
Farm area & 3.46 & 1.08 & 11.653 & $0.0000^{* * *}$ \\
Average meals per day & 2.73 & 2.58 & 2.2610 & $0.0243^{* *}$ \\
\hline
\end{tabular}

Source: own survey, $* * *$ and $* *$, indicates that at $1 \%$ and $5 \%$ significance level respectively

The descriptive and inferential statistics results presented in Table 1 show that $67.6 \%$ of non- users were male headed households. From users of irrigation only $20 \%$ of household were female headed households. Regarding to owning bank account in the sample households only $5 \%$ of users and $0.8 \%$ of non-users have bank account which shows the culture of saving is very low in the study area. Participation in public work was one variable involved in study from the result small portion (20\% of users and 3.2\% non- users) of the respondents have active participation in public work. Getting extension service was the important variable in the study area and significant at $1 \%$ as shown in table 2.

Table 2. Descriptive statistics of Dummy/ discrete Independent Variables

\section{Variables}

Sex of household head

-Male

-Female

Owning bank account

Yes

No

Remittance

Yes

No

public work

Yes

No

Extension visit

Yes

No

\section{Percentage of adoption category}

\begin{tabular}{llll} 
User & Non-user & $\chi 2$ value & p-value \\
\hline 12 & 111 & 3.6799 & $0.055^{* *}$ \\
48 & 232 & & \\
3 & 3 & & \\
57 & 340 & 5.92 & $0.015^{* *}$ \\
3 & 6 & & \\
57 & 337 & 2.4716 & 0.116 \\
12 & 11 & & \\
48 & 332 & 26.76 & $0.000^{* * *}$ \\
50 & 147 & & \\
10 & 196 & 33.48 & $0.000^{* * *}$
\end{tabular}

Source: own survey, ${ }^{* * *}$ and $* *$, indicates that at $1 \%$ and $5 \%$ significance level respectively

\section{Econometric model}

This section presents the results of the probit regression model which is used to estimate propensity scores for matching adopted households with non-adopters. As indicated earlier, the dependent variable in this model is binary variable indicating whether the household uses irrigation or not and the outcome variable is food security index of the house hold head. In the estimation, data from the two groups; namely, users and non- users households were pooled such that the dependent variable takes a value 1 if the household uses irrigation and 0 otherwise.

Table 3 shows the estimation results of the logit model. The estimated model appears to perform well for our intended matching exercise. The pseudo- $\mathrm{R}^{2}$ value is 0.4571 , which is fairly low. A low pseudo- $\mathrm{R}^{2}$ value means that household uses irrigation do not have many distinct characteristics overall and as such finding a good match between users of irrigation and non-users of irrigation becomes easier, and the pseudo- $\mathrm{R}^{2}$ indicates how well independent variables explain the probability of using irrigation. 


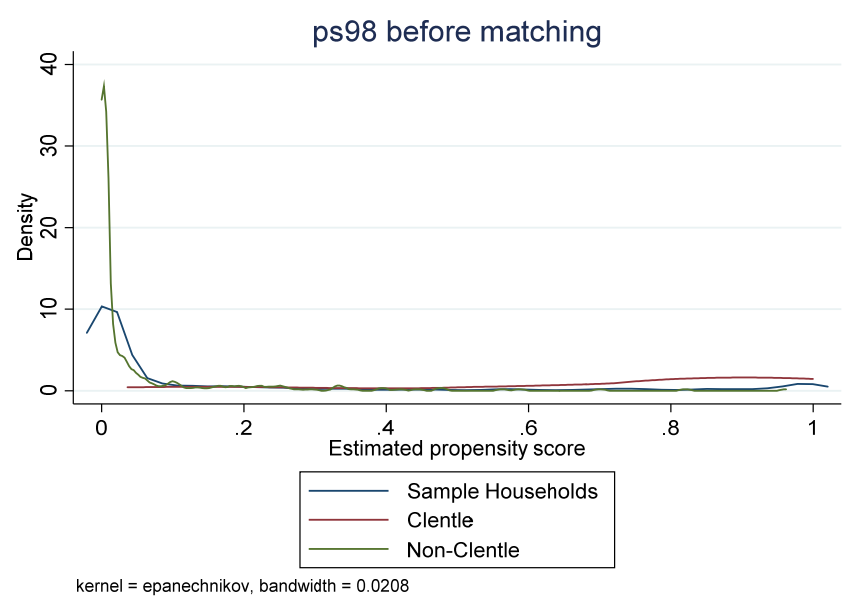

Figure 1. Kernel density of propensity score distribution before matching Source: own sketch (2020)

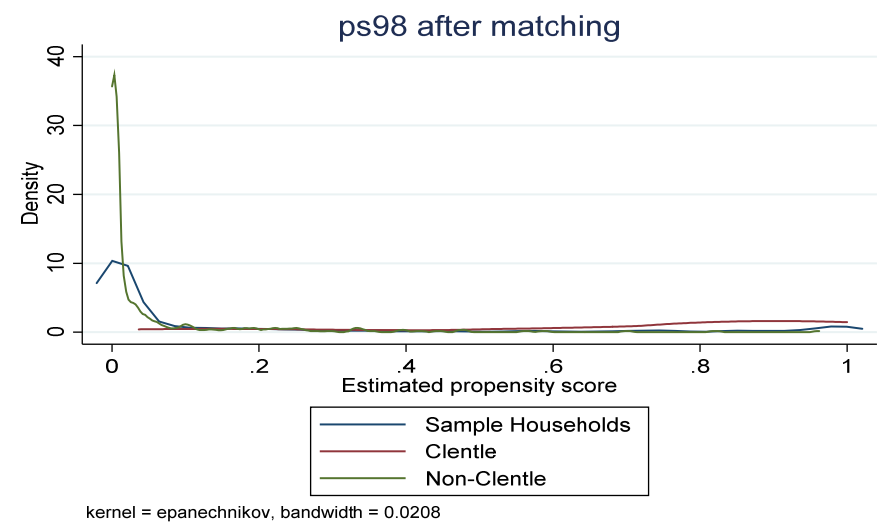

Figure 2. Kernel density of propensity score distribution after matching Source: own sketch (2020)

Probit regression

$$
\begin{array}{llll}
\text { Probit regression } & \text { Number of obs } & =403 \\
& \text { LR chi2 }(15) & = & 225.05 \\
& \text { Prob }>\text { chi2 } & = & 0.0000 \\
\text { Log likelihood }=-57.043566 & \text { Pseudo R2 } & =0.0636
\end{array}
$$

Table 3. Logit model

\begin{tabular}{lcccccc}
\hline irr & Coef. & Std.Err. & $\mathrm{Z}$ & $\mathrm{P}>\mathrm{z}$ & {$[95 \%$ Conf. } & Interval] \\
\hline area & 0.425 & 0.098 & 4.310 & $0.000^{* * *}$ & 0.232 & 0.618 \\
hhsize & -0.153 & 0.073 & -2.100 & $0.036^{* *}$ & -0.295 & -0.010 \\
age & -0.039 & 0.014 & -2.830 & $0.005^{* * *}$ & -0.066 & -0.012 \\
rcpc & 0.014 & 0.007 & 1.970 & $0.049 * *$ & 0.000 & 0.028 \\
poor & 0.611 & 0.479 & 1.270 & 0.202 & -0.328 & 1.550 \\
credit & 0.428 & 0.407 & 1.050 & 0.293 & -0.369 & 1.225 \\
fert & -1.430 & 0.329 & -4.350 & $0.000^{* * *}$ & -2.074 & -0.786 \\
meals & 0.204 & 0.315 & 0.650 & 0.518 & -0.414 & 0.821 \\
off_farm & -0.287 & 0.293 & -0.980 & 0.328 & -0.861 & 0.288 \\
rem & -1.295 & 0.298 & -4.340 & $0.000^{* * *}$ & -1.880 & -0.710 \\
shortage_ox & 0.112 & 0.354 & 0.320 & 0.752 & -0.582 & 0.806 \\
ext & 0.692 & 0.309 & 2.240 & $0.025 * *$ & 0.086 & 1.297 \\
water_h & 1.168 & 0.475 & 2.460 & $0.014 * *$ & 0.237 & 2.098 \\
TLU & 0.256 & 0.073 & 3.500 & $0.000^{* * *}$ & 0.113 & 0.400 \\
yr_schooling & -0.041 & 0.051 & -0.790 & 0.428 & -0.141 & 0.060 \\
cons & -0.702 & 1.248 & -0.560 & 0.574 & -3.149 & 1.745
\end{tabular}

Note: 0 failures and 1 success completely determined. 


\subsubsection{Matching users and non-users of small scale irrigation households}

The common support region would then lies between 0.01875991 and 0.9999822 that is the minimum and the maximum value of treated and control households, respectively. This ensures that any combination of characteristics observed in the treatment group can also be observed among the control group. In other words, households whose estimated propensity scores are less than 0.01875991 and larger than 0.9999822 are not considered for matching exercise. This is because no matches can be made to estimate the average treatment effects on the ATT parameter when there is no overlap between the treatment and non-treatment group (Bryson et al., 2002). As a result of this restriction, 210 households from the control were discarded.

Table 4.Region of common support

\begin{tabular}{llllll}
\hline Variable & Obs & Mean & Std. Dev & Min & Max \\
\hline Ps98 if if $=1$ & 60 & 0.6174 & 0.3155 & .01875 & 0.9999 \\
Ps98 if if $=1$ & 133 & 0.0645 & 0.1443 & $1.36 \mathrm{e}-06$ & 0.9472 \\
Ps98 & 403 & 0.14689 & 0.2668 & $1.36 \mathrm{e}-06$ & 0.99998 \\
\hline
\end{tabular}

Source: Own estimation (2016)

Table 5. Chi-square test for the joint significance of variables

\begin{tabular}{llll}
\hline Sample & Pseudo R & LR chi & \\
\hline Unmatched & 0.475 & 84.90 & P chi $^{2}$ \\
Matched & 0.222 & 8.20 & 0.000 \\
\hline
\end{tabular}

Source: own computation (2016)

\subsubsection{Treatment effect on the treated (ATT)}

This sub-section provides evidence as to whether or not the adoption of improved soybean variety has brought significant changes on farm income. The radius caliper estimator with band width 0.1 , the best matching estimator for the data at hand, was used to compute the average impact of improved soybean variety among adopter households.

Table 6.Average treatment effect on the treated (ATT)

\begin{tabular}{crrrrr}
\hline Variable $($ birr $)$ & Treated & Control & \multicolumn{1}{c}{ ATT } & \multicolumn{1}{l}{ SE } & \multicolumn{2}{c}{ T-value } \\
\hline Food Index KM(0.25) & 333.699 & 333.087 & 0.6117 & 1.391 & 0.44 \\
Food Index caliper(0.01) & 333.699 & 335.040 & -1.341 & 3.281 & -0.41 \\
\hline
\end{tabular}

Note: $\mathrm{SE}=$ Bootstrapped standard errors with 100 replications;

\section{SUMMARY, CONCLUSIONS AND RECOMMENDATIONS}

\subsection{Summary and Conclusions}

Ethiopia is one of the poorest countries in the world, where about $29.2 \%$ of its population live below poverty line (World Bank, 2013). The county is endowed with natural resource which is highly suitable for agricultural production but dependence on traditional and rain feed agriculture results poor yield. This results starvation and poverty in the country. It plays great role to be food insecure.

This study was conducted in SNNP region with 60 small scale irrigation users and 343 non-users. We have used PSM model to match users and non-users.

Southern Nations and Nationalities (SNNP) Region is highly endowed with natural resource high rain relative to other regions. Based on this study application of irrigation in this region does not have impact on yield by using different matching allogarisms. Therefore, it is better to switch irrigation activities in other regions by which getting rain is relatively low.

\section{REFERENCES}

Bryson, R Dorsett, S Purdon. (2002). the use of propensity score matching in the evaluation of active labour market policies

Caliendo and Kopeing (2008). Some practical guidance for the implementation of propensity score matching

Dawid (1979). Conditional independence in statistical theory.

Dehejia Wahba (1999). Causal effects in nonexperimental studies: Reevaluating the evaluation of training programs

Fisher (1925). The influence of rainfall on the yield of wheat at Roth Amsted

Imbems (2004). Implementing matching estimators for average treatment effects in Stata.

Neyman (1923). The application of probability theory to agricultural experiments. Essay on principles. Section 9.

Rubin (1974).Statistical techniques for analyzing data from prevention trials: Treatment of no-shows using Rubin's causal model 


\section{Appendix 1.}
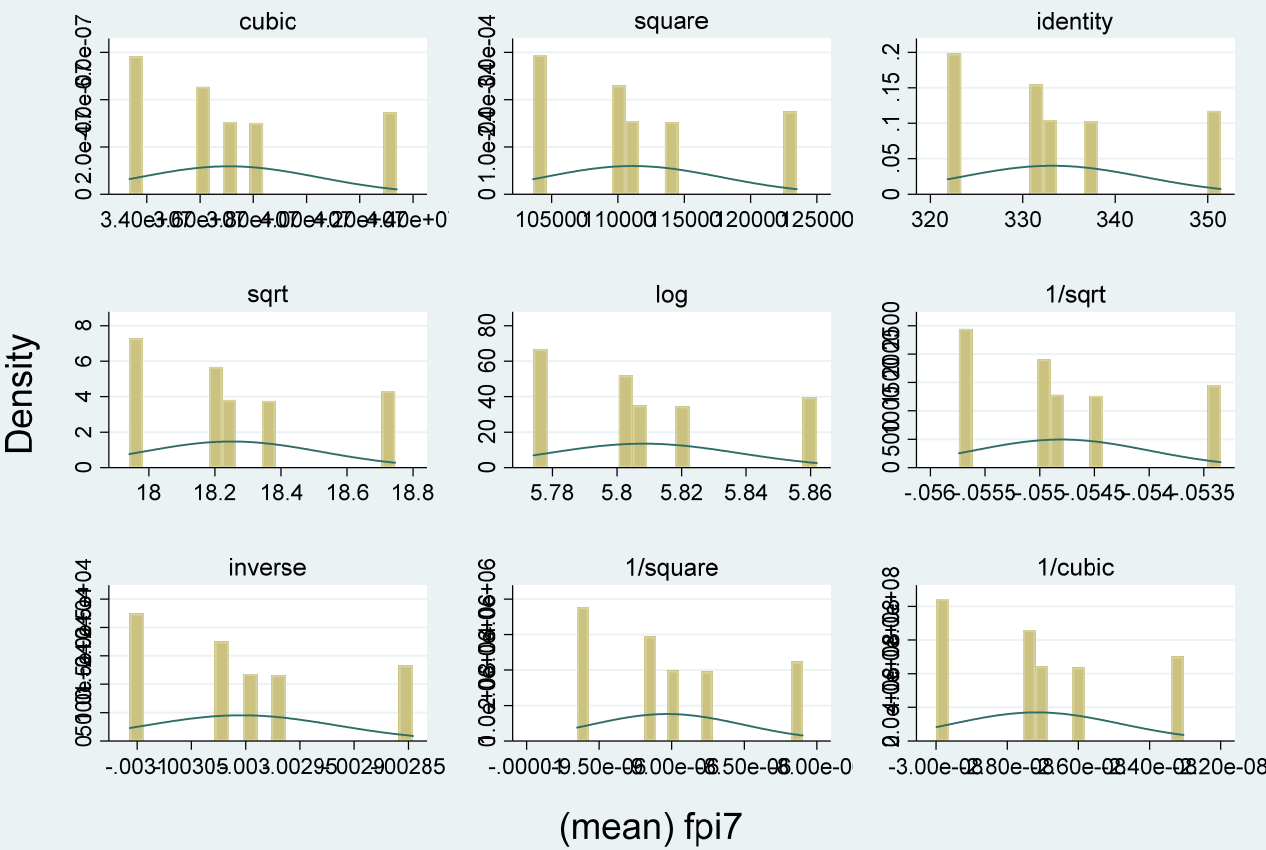

Histograms by transformation

Figure 3. Graph for normality checking (gladder sintax) 